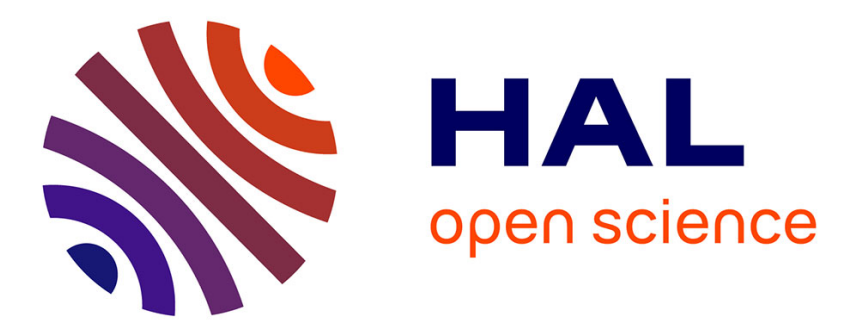

\title{
(Se) convertir : les ressorts de la transformation biographique
}

\author{
Gabrielle Angey, Yannick Fer, Martial Vildard
}

\section{To cite this version:}

Gabrielle Angey, Yannick Fer, Martial Vildard. (Se) convertir: les ressorts de la transformation biographique. Genèses. Sciences sociales et histoire, 2021, 124, pp.3-7. halshs-03471028

\section{HAL Id: halshs-03471028 \\ https://shs.hal.science/halshs-03471028}

Submitted on 8 Dec 2021

HAL is a multi-disciplinary open access archive for the deposit and dissemination of scientific research documents, whether they are published or not. The documents may come from teaching and research institutions in France or abroad, or from public or private research centers.
L'archive ouverte pluridisciplinaire HAL, est destinée au dépôt et à la diffusion de documents scientifiques de niveau recherche, publiés ou non, émanant des établissements d'enseignement et de recherche français ou étrangers, des laboratoires publics ou privés. 


\section{(Se) convertir : les ressorts de la transformation biographique}

Gabrielle Angey, Yannick Fer et Martial Vildard

Les représentations ordinaires de la conversion oscillent entre un discours de l'émancipation, qui souligne la capacité de choisir par soi-même une identité et une inquiétude face aux mécanismes d'emprise susceptibles d'éloigner radicalement des individus de leurs appartenances d'origine. Dans le premier cas, l'accent est mis sur l'autonomie individuelle ; dans le second, sur la force des structures et des relations d'autorité qui produisent la transformation biographique.

Le point de vue des sciences sociales consiste a priori à penser ensemble l'expérience subjective de la liberté et le jeu des structures sociales. Toutefois, la littérature scientifique sur la conversion religieuse - qui est foisonnante - insiste bien plus souvent sur sa dimension individualisante que sur son imbrication avec d'autres rapports sociaux. L'attention se porte principalement sur les facteurs de la conversion, en amont, et sur ses effets individuels et sociaux, en aval. La nature précise du processus de transformation biographique est plus rarement explorée. L'omniprésence du récit de conversion peut même conduire à assimiler les mécanismes de transformation des dispositions personnelles à un discours performatif et la conversion à un fait de langage. La dimension institutionnelle réapparaît surtout dans les contextes religieux où le changement d'appartenance est soumis à une procédure formelle d'évaluation et de validation, comme dans le judaïsme (Tank-Storper, 2007).

Et pourtant, nous savons depuis Émile Durkheim que ce «mouvement profond par lequel l'âme tout entière, se tournant dans une direction toute nouvelle, change de position, d'assiette et modifie, par suite, son point de vue sur le monde » implique un travail d'éducation et de (re)socialisation (Durkheim, 1990 [1938] : 38). La conversion n'est donc pas simplement une scène où le social s'efface au profit d'une identité librement façonnée par la conviction religieuse, mais plutôt un processus relationnel, qui met en jeu tout autant l'individu que des communautés et des institutions. Particulièrement prégnante en sciences sociales des religions, l'idée d'une vie sociale et religieuse qui serait de plus en plus marquée par un double processus d'individualisation 
et de désinstitutionnalisation [4] conduit de fait à présumer à tort une opposition de principe entre l'autonomisation des trajectoires personnelles et l'encadrement institutionnel des existences. Il s'agit ici de les penser ensemble.

Ce dossier s'intéresse aux conditions dans lesquelles des individus sont amenés à changer, à intérioriser durablement de nouvelles dispositions, de nouvelles normes morales, et à revendiquer un changement d'identité significatif. Il appréhende cette conversion comme une mesure de l'autonomie individuelle et de ses conditions sociales (Geay, 2009), en replaçant au cœur de l'analyse la socialisation et le rôle de l'institution comprise dans sa dimension objectivée et incorporée. La dimension objectivée renvoie à des dispositifs d'encadrement, d'éducation et de socialisation. La dimension incorporée rappelle quant à elle le rôle des structures mentales qui fondent l'existence de l'institution dans les esprits et les corps (Bourdieu 2015: 236), mais aussi les potentiels désajustements susceptibles d'apparaître notamment au gré d'évènements affectant individus ou institutions. Cette dimension incorporée souligne en outre, dans une perspective plus interactionniste, l'importance du groupe d'appartenance comme espace de socialisation où la conversion peut être élaborée collectivement et reconnue (Heurtin et Michel, 2021 : 7), sur le mode intime d'un partage d'expériences.

Le rapport entre dispositions personnelles et dispositifs institutionnels n'est donc pas donné d'emblée, ni mécanique. Il passe par une série de médiations dont la sociologie doit rendre compte, à distance des grands récits en surplomb. C'est pourquoi les articles de ce dossier s'appuient sur des enquêtes de terrain extensives et une ethnographie rapprochée, lesquelles aident à repérer les points d'articulation entre travail sur soi, socialisation et prise en charge institutionnelle, notamment en introduisant dans l'analyse le rôle des socialisations antérieures. Employer la sociologie des institutions pour rendre compte de ces conversions permet d'en restituer la dimension processuelle, en inscrivant dans le temps long l'ensemble des efforts consentis en vue de faire de la conversion une "illusion bien fondée ", c'est-à-dire produire des changements biographiques significatifs et durables (Darmon, 2011 : 75), ou les préserver face à des contextes défavorables. La notion de travail institutionnel occupe une place centrale dans ce dossier. Elle nous conduit à réaffirmer l'intérêt d'une sociologie des formes de l'autorité sur les terrains d'enquête religieux, contre la thèse de l'avènement contemporain d'une « autorité personnelle » (self-authority) synonyme de disparition des 
rapports de pouvoir. Dès 1983, James Beckford soulignait que la notion de pouvoir est de nature à constituer un pont heuristique entre la sociologie des religions et l'analyse sociologique plus générale des structures et des processus sociaux (Beckford, 1983 : 29). Notre dossier s'inscrit dans une perspective similaire. Mais la sociologie des conversions religieuses doit, dans le même temps, accorder sa juste place à l'événement : si l'observation des processus de resocialisation invite à relativiser le récit de la conversion comme événement au profit d'une analyse plus processuelle, nos enquêtes interrogent aussi à plusieurs reprises la capacité de certains [5] événements à susciter, selon les circonstances sociales et personnelles dans lesquelles ils surviennent, une transformation biographique radicale (Pagis, 2014: 97). Cette double perspective traverse l'ensemble des articles de ce dossier.

À partir de ses enquêtes sur les «nouvelles spiritualités », Matthew Wood a montré comment l'insistance sur « l'autorité personnelle » empêche de saisir l'influence persistante des autorités religieuses sur les dispositions personnelles des croyants : l'autorité n'y disparaît pas, mais parce que les individus s'en remettent simultanément à plusieurs autorités, l'effet structurant de chacune d'entre elles se trouve relativisé (Wood, 2021). Les articles de ce dossier indiquent de quelles manières la relation d'autorité peut être reformulée, afin d'accompagner l'individualisation des pratiques religieuses et de conforter la croyance en une authenticité personnelle comprise comme synonyme de déprise institutionnelle. Juliette Galonnier décrit dans le cas des associations prenant en charge les parcours de conversion à l'islam des formes d'autorité amicale conjuguant des échanges en petits groupes et le maintien d'une orthodoxie par le biais d'une apparente neutralité pédagogique. Pareille combinaison, mêlant création d'espaces de socialisation entre pairs, informalisation des rapports d'autorité et recours à la pédagogie s'observe dans plusieurs autres contextes. Au sein des églises pentecôtistes étudiées par Yannick Fer, les groupes de «formation à la vie chrétienne » permettent d'inscrire l'effort de réformation de soi dans un cadre où le travail institutionnel se rend invisible, sous la forme d'une double relation de compagnonnage (entre convertis) et d'accompagnement (par un mentor). C'est aussi sur les sohbet, petits groupes de discussion et d'étude, que repose pour une bonne part la cohésion du mouvement Gülen. C'est là, explique Gabrielle Angey, que ses membres incorporent des dispositions à l'engagement et qu'ils trouvent refuge en dernière instance lorsque le versant objectivé de l'institution à laquelle ils 
appartiennent vacille : au sein de cet espace intermédiaire, ils remobilisent alors un ensemble de ressources institutionnelles face à l'adversité.

On se tromperait donc en ne voyant dans ces petits groupes que la simple substitution d'une socialisation horizontale à un encadrement institutionnel. Ils indiquent au contraire les voies par lesquelles l'autorité institutionnelle se reconfigure pour maintenir sa capacité à transformer les existences personnelles. Cette reconfiguration ne concerne pas seulement les religions peu cléricalisées, comme l'islam, ou celles qui revendiquent le primat de la «relation personnelle avec Dieu » sur l'appartenance institutionnelle, comme le pentecôtisme. Le paradigme de l'accompagnement personnalisé inspire aujourd'hui toute une série de dispositifs de canalisation des expériences individuelles et de mise en ordre du monde social. Ces dispositifs se développent partout où il était jusqu'alors question de «formation » et de «prise en charge » : reconversion professionnelle, éducation de la jeunesse, initiation religieuse, assistance sociale ou accompagnement thérapeutique. Ils se déclinent aussi bien sur le mode de la « relation d'aide » évangélique que dans le cadre du catéchuménat catholique des adultes, qui mobilise des [6] accompagnants choisis et formés par l'institution pour inscrire la conversion dans le cadre enchanté d'une « rencontre avec le Christ» (Vildard, 2015). Au fil de ces reconfigurations, l'autorité se maintient en même temps qu'elle se transforme. Ce constat conduit à explorer la manière dont l'autonomie individuelle travaille l'institution et, en retour, les mécanismes par lesquels l'institution continue d'opérer comme une instance légitime de resocialisation.

Dans ce jeu complexe entre individualisation, resocialisation et institution demeure toujours la possibilité de l'inattendu, d'une échappée au moins relative hors des déterminations sociales objectives. Si la conversion requiert en effet une série de prédispositions, les conditions d'actualisation de celles-ci sont en partie le fruit des circonstances. Ce dossier conjugue ainsi une approche attentive aux processus sur le temps long et une attention à l'évènement. Celui-ci ne se situe pas nécessairement où il est attendu dans les conversions religieuses. C'est notamment l'idée selon laquelle la conversion religieuse déboucherait logiquement sur une rupture biographique et sur l'acquisition de dispositions inédites, qui est examinée à nouveau frais.

À travers son enquête dans les églises pentecôtistes de Polynésie française et sur le réseau charismatique Youth With a Mission, Yannick Fer montre comment l'institution 
et le converti coconstruisent la trame des événements nécessaires à l'expérience - et au récit - de conversion. Aux ruptures biographiques qui prédisposent à rechercher une solution à la fois radicale et extraordinaire répondent des dispositifs institutionnels visant à produire la rencontre inattendue «avec Dieu » comme point de départ d'un parcours de réinvention. Encastré dans des dispositifs institutionnels, ce récit de soi accompagne comme un appui nécessaire le processus lent d'alternation auquel se soumet le croyant par le travail sur soi. Dans le cas des convertis musulmans, le changement d'affiliation religieuse est bien en lui-même un événement qui s'oppose visiblement au poids des institutions sociales. Les associations de convertis travaillent dès lors, tout en préservant l'expérience subjective de l'événement, à réduire la portée des dissonances et des incidences personnelles de cette rupture biographique pour rendre la conversion pérenne.

Deux articles de ce dossier proposent quant à eux une approche originale des liens entre rupture biographique et conversion des dispositions : ils montrent que le changement lié à un événement provoquant une rupture biographique n'est pas toujours synonyme d'inédit. Thibault Ducloux a suivi des détenus sur le temps long, de leur entrée en prison jusqu'à l' « illumination spirituelle » dont plusieurs font l'expérience. Gabrielle Angey a rencontré en Afrique de l'Est des fidèles musulmans originaires de Turquie passés de force à la clandestinité en raison de leur appartenance au mouvement Gülen réprimé par le gouvernement turc qui les accuse de terrorisme. Dans ces deux cas, les auteurs ne font pas découler mécaniquement l'éventuelle conversion des dispositions de l'entrée en religion mais examinent la portée d'événements dramatiques (entrée en prison, passage à la clandestinité et exil) qui suspendent les modalités routinières de mise en œuvre de l'habitus au [7] quotidien. Ils montrent qu'en situation de rupture biographique consécutive à un événement subi, la conversion consiste moins en l'incorporation d'un nouvel habitus qu'en une régression vers des prédispositions latentes. Dans le contexte d'une institution totale comme celle de la prison, le système de dispositions construit par les socialisations secondaires (réseaux de banditisme, vie familiale) ne peut plus fonctionner. Pour leur survie psychique, certains individus s'en remettent alors de manière non-consciente à une socialisation régressive, renouant avec des pratiques religieuses de leurs familles dont ils s'étaient auparavant éloignés. Exilés en Afrique de l'Est, les fidèles musulmans voient leurs systèmes de dispositions, produit 
d'une socialisation longue au sein de l'institution Gülen, menacés. Par un travail sur euxmêmes, encore encadré par une institution religieuse elle-même affaiblie, et avec un zèle plus ou moins fort selon leurs trajectoires sociales, les croyants travaillent à maintenir leurs dispositions pour éviter de perdre pied. La régression vers des dispositions incorporées antérieurement apparaît ainsi dans sa dimension laborieuse. La réponse à des contextes de crise et à l'effondrement des cadres routiniers d'existence produit donc un changement biographique décisif, mais ce qui est nouveau d'un point de vue religieux et subjectif n'est pas à proprement parler inédit.

Ce dossier souligne finalement la manière dont la notion de conversion, saisie à partir d'une sociologie de la socialisation et de l'institution, peut contribuer à une analyse plus générale des changements personnels et enrichir la compréhension des conditions sociales de l'autonomie individuelle. En inscrivant la sociologie des conversions religieuses dans cette perspective théorique, notre intention n'est pas d'étendre l'analogie religieuse à d'autres sphères du social, mais au contraire de réinscrire le religieux dans le social et l'étude du religieux dans les débats des sciences sociales (Altglas et Wood, 2018). En l'occurrence, les cas religieux de transformation biographique rappellent, sans doute plus nettement que d'autres, la nécessité pour la sociologie de prendre au sérieux l'expérience individuelle du changement et les récits qui l'accompagnent, sans pour autant les prendre au pied de la lettre.

\section{Bibliographie}

Altglas, Véronique et Wood, Matthew Wood (dir.). 2018. Bringing Back the Social into the Sociology of Religion. Critical Approaches. Leiden, Brill.

BECKFORD, James. 1983. "The restoration of 'Power' to the Sociology of Religion", Sociological analysis 44(1) : 11-32.

BouRdiEU, Pierre. 2015. Sociologie générale, vol. 1. Paris, Seuil.

DARMON, Muriel. 2011. "Sociologie de la conversion : socialisation et transformations individuelles", in Claudine Burton-Jeangros et Christoph Maeder (dir.). Identité et transformation des modes de vie. Zurich, Seismo : 64-84.

DuRKHEIM, Émile. 1990 (1938). L'évolution pédagogique en France. Paris, PUF. GEAY, Bertrand. 2009. "L'impératif d'autonomie... et ses conditions sociales de production », in Marlène Jouan et Sandra Laugier (dir.), Comment penser l'autonomie ? Paris, PUF : 151-168. 
HEURTIN, Jean-Philippe et Michel, Patrick (dir.). 2021. La conversion et ses convertis. Production et énonciation du changement individuel dans le monde contemporain. Paris, Politika.

LAGROYE, Jacques. 2009. Appartenir à une institution : Catholique en France aujourd'hui. Paris, Economica.

PaGis, Julie. 2015. Mai 68, un pavé dans leur histoire. Paris, Presses de Sciences Po.

TANK-STORPER, Sébastien. 2007. Juifs d'élection. Se convertir au judaïsme. Paris, CNRS éditions.

VILDARD, Martial. 2015. «L'accompagnement personnalisé des candidats au baptême de l'Église catholique dans le diocèse de Paris. Étude sociologique d'un dispositif de transmission des normes institutionnelles ». Mémoire de master "Sciences des religions et société ». Paris, EPHE.

Wood, Matthew. 2021. Spiritualité et pouvoir. Les ambiguïtés de l'autorité religieuse. Genève, Labor et Fides. 